\title{
An Analysis of Time-Series Models for Age-Specific Mortality Rates in India
}

\author{
Aritra Sen $^{1}$ \\ ${ }^{1}$ Department of Biostatistics and Demography \\ ${ }^{1}$ International Institute for Population Sciences \\ Mumbai, India.
}

\begin{abstract}
Mortality is a continuous force of attrition, tending to reduce the population, a prime negative force in the balance of vital processes (Bhasin and Nag, 2004). Sample Registration System (SRS) serves as the only source of annual data on vital events on a full scale from 1969-70 in India. Few studies have examined the trends and patterns of mortality across time and regions in India (Preston and Bhat, 1984). The Under 5 Mortality Rates (U5MR) can be seen to decrease by more than half from 1970 to 2017 but in contrast little is known about the mortality patterns of the older children (5-9) and young adolescents (10-14), and not many studies have been done on their changing trends (Masquelier et al., 2018).
\end{abstract}

Using the annual data for the 5-14 age, the trend of decline in the mortality patterns is studied from 1970 to 2013. The linear trend in the time series plot suggests analysis using time series models $\operatorname{AR}(p), \operatorname{MA}(q)$, $\operatorname{ARMA}(p, q)$, Box- Jenkins $\operatorname{ARIMA}(p, d, q)$ and Random Walk with drift models to get the best fit to the trend of the data. The order of the time series models have been calculated by studying the ACF, PACF plots and the coefficients have been derived using the Yule-Walker equation matrix. An in-sample forecast of the years 2014-17 are taken. The Mean Squared Error (MSE) and the Mean Absolute Percentage Error (MAPE) as a measure of accuracy is used to determine the best fit model. ARIMA $(3,1,1)$ produced lower values making it the best-fit model.

Out-of-sample forecasting was done for 2018-2025. The forecast value shows that at the current trend, India would have 0.03 deaths per 1000 population in the 5-14 age group in 2025 showing that the government's policies and health care interventions towards realization of the MDG4 goal is working positively.

Keywords:- ARIMA; yule-walker; SRS; MAPE; MSE

\section{INTRODUCTION}

Mortality Rate is a measure of the number of deaths (in general, or due to a specific cause) in a particular population, scaled to the size of that population, per unit of time, typically expressed in units of deaths per 1,000 individuals per year. Among the components of population change, mortality plays a vital role in determining the size and influencing the age-structure of the population to certain extent. Indirect estimates by

\author{
Shalmoli Dutta ${ }^{2}$ \\ ${ }^{2}$ Department of Biostatistics and Demography \\ ${ }^{2}$ International Institute for Population Sciences \\ Mumbai, India.
}

World Health Organization (WHO) and the Global Burden of Diseases Study (GBD) suggest that unintentional injuries account for 3.9 million deaths worldwide (WHO, 2008). Registration of births and deaths is an important source for demographic data for socioeconomic development and population control in developing countries.

Sample Registration System (SRS) serving as the only source of annual data on vital events on a full scale from 1969-70 marks the decline in Overall Crude Death Rate (CDR) from 15.7 in 1970 to 6.3 in 2017. The Infant Mortality rate (IMR) which is an important indicator of health status in the country has declined as well from 129 to 33 in the same period. At urban level, the CDR changed to 5.3 and in rural it has come down to 6.9. Several public health policies through time had varied implications in the declining mortality rates of women specifically, that can be seen to be 4.9 for urban female and 6.4 for rural in 2017. It is noteworthy that the CDR for female came to be lower than male for both urban and rural (Chaurasia, 2006). From 1970 to 2016 , the mortality of children younger than 5 years decreased by more than half, and there are plenty of data regarding mortality in this age group through which we can track global progress in reducing the under-5 mortality rate. By contrast, little is known on how the mortality risk among older children (5-9 years) and young adolescents (10-14 years) has changed in this time (Masquelier et al., 2018). This neglect is presumably associated with the fact that this is the age range in which the risk of mortality is lowest (Mathers, 2015).

Three approaches can be outlined in studying and analyzing the trends in age patterns of mortality namely the Analytical (mathematical) approach, Empirical approach and Relational approach (Ewbank et al., 1983). Usually, analytical approach refers a parametric function $f\left(x ; a_{1}, a_{2}, \ldots . a_{n}\right)$ where $\mathrm{x}$ is the age variable and $a_{1}, a_{2}$, $\ldots . a_{n}$ are the parameters that provide a close estimate to a class of mortality experience. Heligman and Pollard (1980) proposed a descriptive "Law of Mortality" depicting the shape of human mortality with an eight-parameter model for all ages. In the empirical approach typical patterns are selected from real life tables and are used to generate models by different level of mortality within each identified pattern known as Model Life Table (MLT) and used for various purposes in circumstances where complete vital registration is not in action (Murray et. al, 2003). Sankrithi et al. (1991), developed a product form multivariate regression model (multiplicative exponential) with infant and child mortality as outcome and national economic, 
health, nutrition, education and demographic statistics as predictor variables with an advantage over sum form models which predicted negative mortalities for some nations. Hussein (1993), used two models, one with the natural logarithmic transformation of infant mortality time series and the other with successive differences to provide infant mortality projections for the period $1983-2000$ in Egypt. Wong et al. (1997), annually analyzed birth and death data of infants in Hong Kong from 1956 to 1990 by aggregating the data into 7 consecutive quinquennia with the aim of developing statistical models to predict changes in the IMR. In a document that presents a Bayesian approach to forecasting mortality rates, an approach that formalizes the Lee- Carter method as a statistical model for all sources of variability, Pedroza (2006), used Markov chain Monte Carlo methods to fit the model and to sample from the posterior predictive distribution. In a work that aimed at comparing the performances of ARIMA, Neural Network and Linear Regression models for the prediction of Infant Mortality Rate, Purwanto et al. (2010), compared the models using performance measures such as Mean Absolute Error (MAE), Mean Absolute Percentage Error (MAPE) and Root Mean Square Error (RMSE), using the Infant Mortality Rate data collected in Indonesia during the years 1995-2008. In a study of predictive cause of death model for U5MR to accelerate the progress towards MDG4, Rao et al. (2010), analyzed over 1400 country years of vital statistics from 34 countries collected over a period of nearly a century, to develop relationships between levels of under-five mortality, related mortality ratios, and proportionate mortality from four cause groups: perinatal conditions, diarrhea and lower respiratory infections; congenital anomalies; and all other causes of death.

Mortality Rate reflects a country's level of socioeconomic development and quality of life and is used for monitoring and evaluating population and health programs and policies. The age group of 5-14 can be seen to present the most variation in time determined by the Inter-Quartile Range, Variance and Coefficient of Variation of the 3-year moving averages of all the age group data. This study will mainly provide a statistical model for predicting the mortality rate for older children (5-9) and young adolescents (10-14) in India at any instant and also bring to the fore, whether or not India is able to achieve her target of significantly reducing the mortality rates. The study will provide policy makers with the evidence and the idea of possible future values of the rates, and thus help them to revise their childhood death intervention strategies so as to maintain and sustain the rates, or to reduce further, if the 2025 rate is found to be far from the target value. All these will ensure that Indian children are healthier and grow to realize their talents and potentials and thus help maintain a strong labour force for the future developmental programs of the nation.

\section{SOURCES OF DATA AND METHODOLOGY}

The increasing acuteness of the population problem in India and deficiencies in the statutory registration system have greatly intensified the need for quick and reliable estimation of fertility and mortality rates on the current and ongoing basis. The Sample Registration System (SRS) in India is the largest demographic survey in the world covering about 1.5 million households and 7.3 million individuals. It has continued to be the main source of information on the vital indicators both at the State and National levels since 1970. The SRS is the most regular source of demographic statistics in India. It is based on a system of dual recording of births and deaths in fairly representative sample units spread all over the country (Mahapatra, 2010). Also, to maintain the representative character of the sample and improve efficiency, the sample units of the SRS are periodically replaced, using the results of the latest population census. A total replacement of the sample has been made twice in the past, once during the years 1982-84, and again during the years 1993-95 (Bhat, 2002). ASDR in 5-year age groups, starting from the 0-4 group till the $70+$ group, have been taken for each year beginning from 1970 till 2017. The 3-year Moving Average data has been taken from the "Compendium of India's Fertility and Mortality Indicators" available in Census India website.

The Box-Jenkins (ARIMA) and the Random Walk with drift methods are used for the analysis. The data available at hand was a non-stationary data which was converted to stationary form to be able to model it. Box and Jenkins (1970) recommends the differencing approach to achieve stationarity. KPSS test and ADF (Augmented Dickey-Fuller) test has been used to confirm stationarity. An Auto-Regressive process with lag of order 'p' can be tried for fitting to begin with. The order of the lag is decided parsimoniously by PACF (Partial Auto Correlation Coefficient) plot of the data to see the number of significant levels. Similarly Moving-average plot is also done with a lag of order ' $q$ '. The order of the lag in this model is decided by the ACF (Auto Correlation Coefficient) plot. The coefficients (weights) of these equations were calculated using the Yule-walker equation matrix. Proceeding further ARMA (Auto Regressive Moving Average) model was fitted of order (p, q) which was estimated earlier. Lastly ARIMA (Auto Regressive Integrated Moving Average) model was used with order ( $\mathrm{p}$, $d, q)$, where ' $d$ ' is the difference operator. The ARIMA procedure analyze and forecasts equally spaced univariate time series data, transfer function data, and intervention data using the Autoregressive Integrated Moving-Average (ARIMA) or autoregressive moving-average (ARMA) model. An ARIMA model predicts a value in a response time series as a linear combination of its own past values and past errors (also called shocks or innovations). Several combinations of ' $p$ ' and ' $q$ ' have been tried to get the model with minimum value of Akaike's Information Criterion (AIC), Akaike's Bias Corrected Information Criterion (AICC) and Bayesian Information Criterion (BIC). Random walk is a process where the current value of 
a variable is composed of the past value plus, an error term defined as a white noise (a normal variable with zero mean and variance one). Algebraically, a random walk is represented as $Y_{t}=Y_{t-1}+\epsilon_{t}$.

\section{RESULTS AND DISCUSSION}

The figure in Figure 1 is a plot of the year wise mortality rate of the 5-14 age group for India from 1970 to 2013, otherwise known as observations and displayed as ordinates against equally spaced time intervals as abscissa, which is used to evaluate the pattern and behavior in the data over time. A visual inspection of the plot shows a downward trend, which may be indicating non-stationarity in the series data.
A confirmation is sought by plotting the correlogram for the time series and shown in the Figure 2(a). The correlogram displays graphically and numerically the autocorrelation function (ACF) and the partial autocorrelation function (PACF) for the time series. The figure shows large significant ACF for the time lags which gradually decreases in size, but do not decay to zero (slow decay). The ACF thus shows a pattern typical of a nonstationary time series. In the PACF plot, the partial autocorrelation at time lag 1 is close to one and the partial autocorrelations for the time lag 2 through 10 are close to zero which is also typical of non-stationary series. The series is transformed by $W_{t}=\Delta y_{t}=y_{t}-y_{t-1}$, in order to stabilize the variance before proceeding with the model building. The ACF and PACF plot for the first differenced series, Figure 2(b) dies out relatively quickly which may be indicating a stationary process.

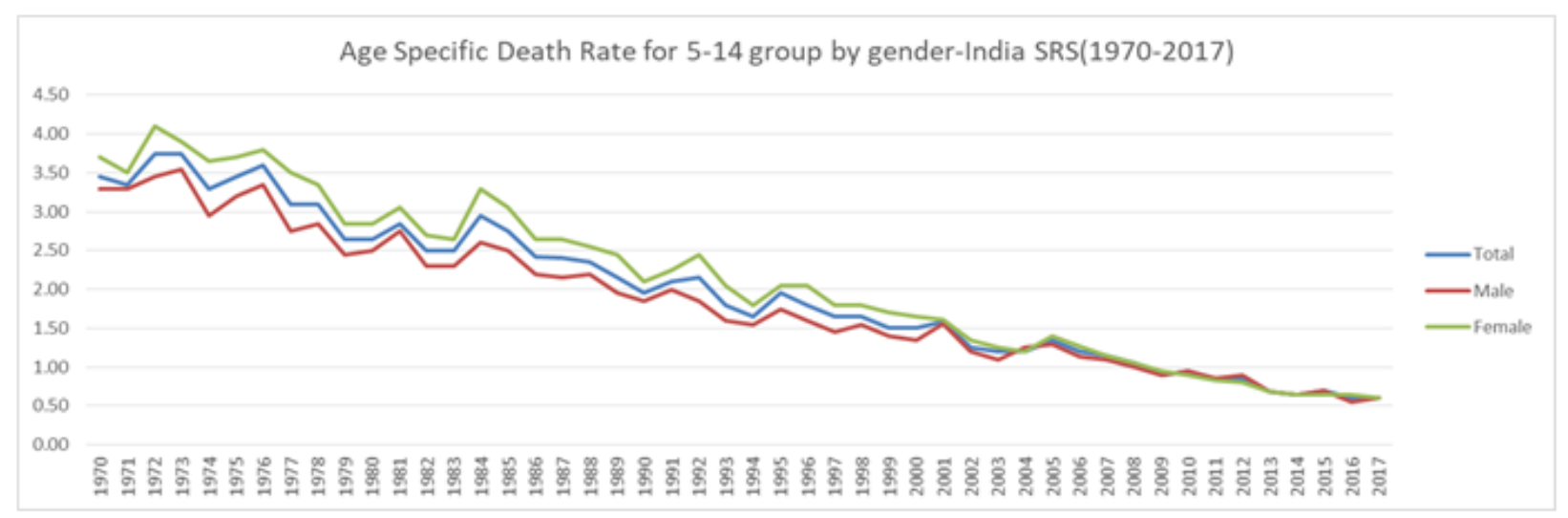

Fig 1:- Trend plot of the 5-14 age mortality rate for India for Combined, Male and Fema

ACF for ASDR of 5-14 group

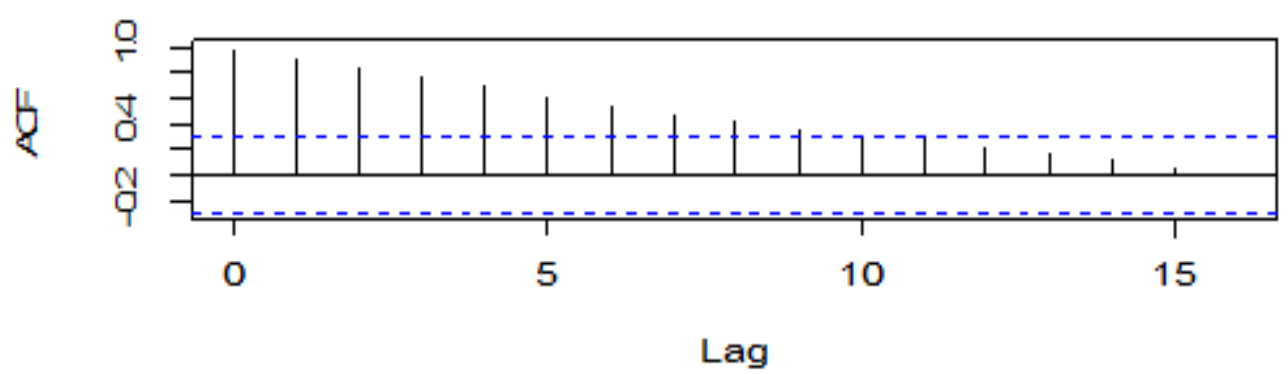

PACF for ASDR of 5-14 group

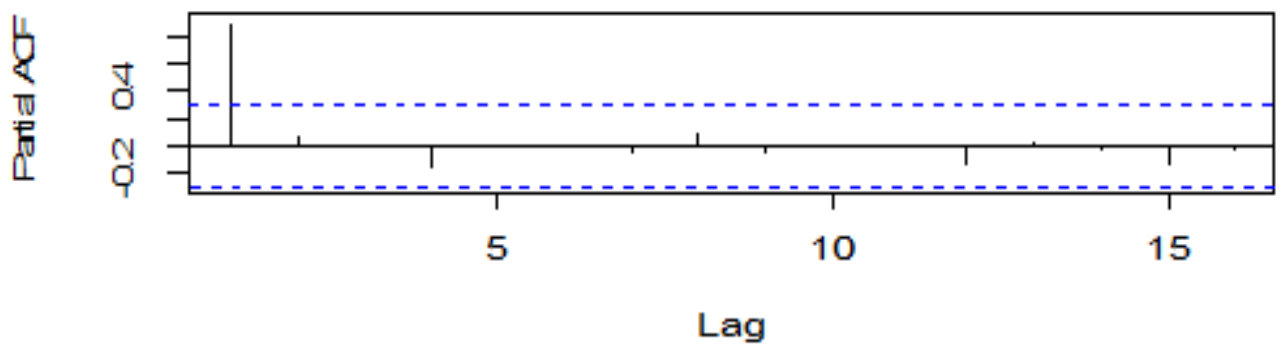

Fig 2(a):- ACF and PACF plot for time series data of total population 
16t difference

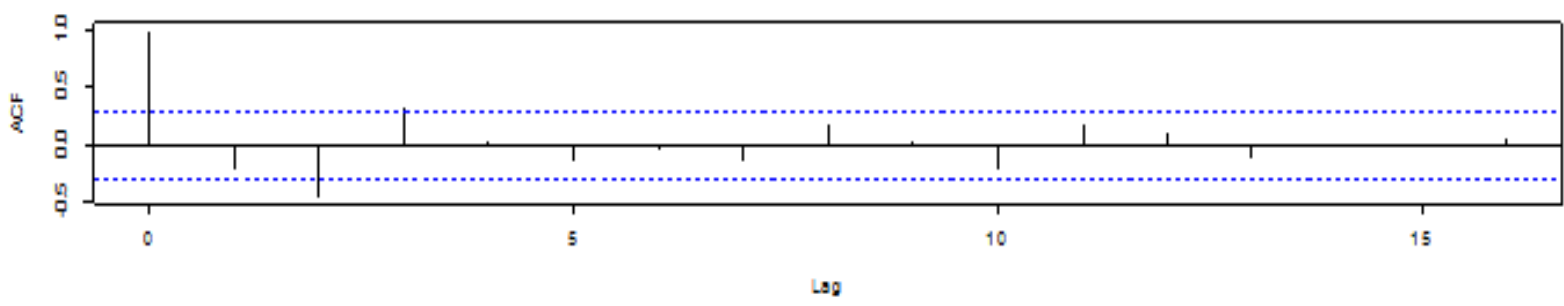

16t difference

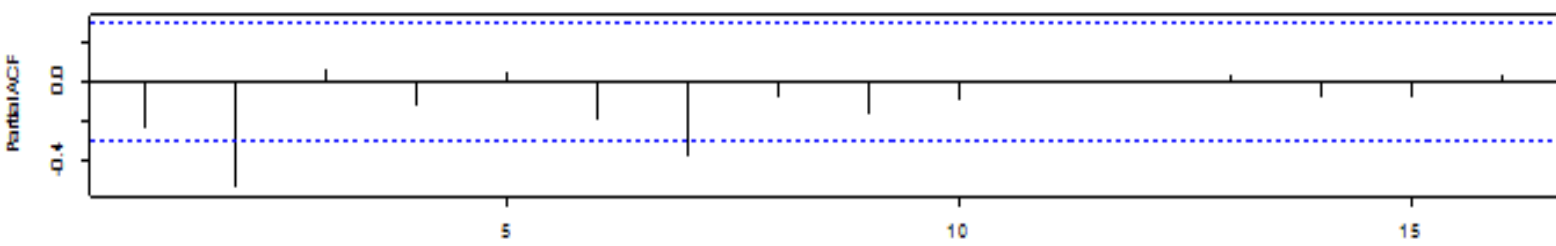

$\Delta g$

Fig 2(b):- ACF and PACF plot for the $1^{\text {st }}$ difference data

In addition to the observed flat level of the first differenced series Figure $2 \odot$, its stationarity is confirmed by the KPSS and (Augmented Dickey-Fuller) ADF tests. The KPSS test statistic with a p-value of 0.1 which is greater than the $5 \%$ level of significance does not reject null hypothesis that the first differenced series is level stationary. The ADF test also produced a pvalue of 0.014 which is less than the 5\% significance level and thus rejects the null hypothesis that the first differenced series is non-stationary, hence confirming the stationarity.
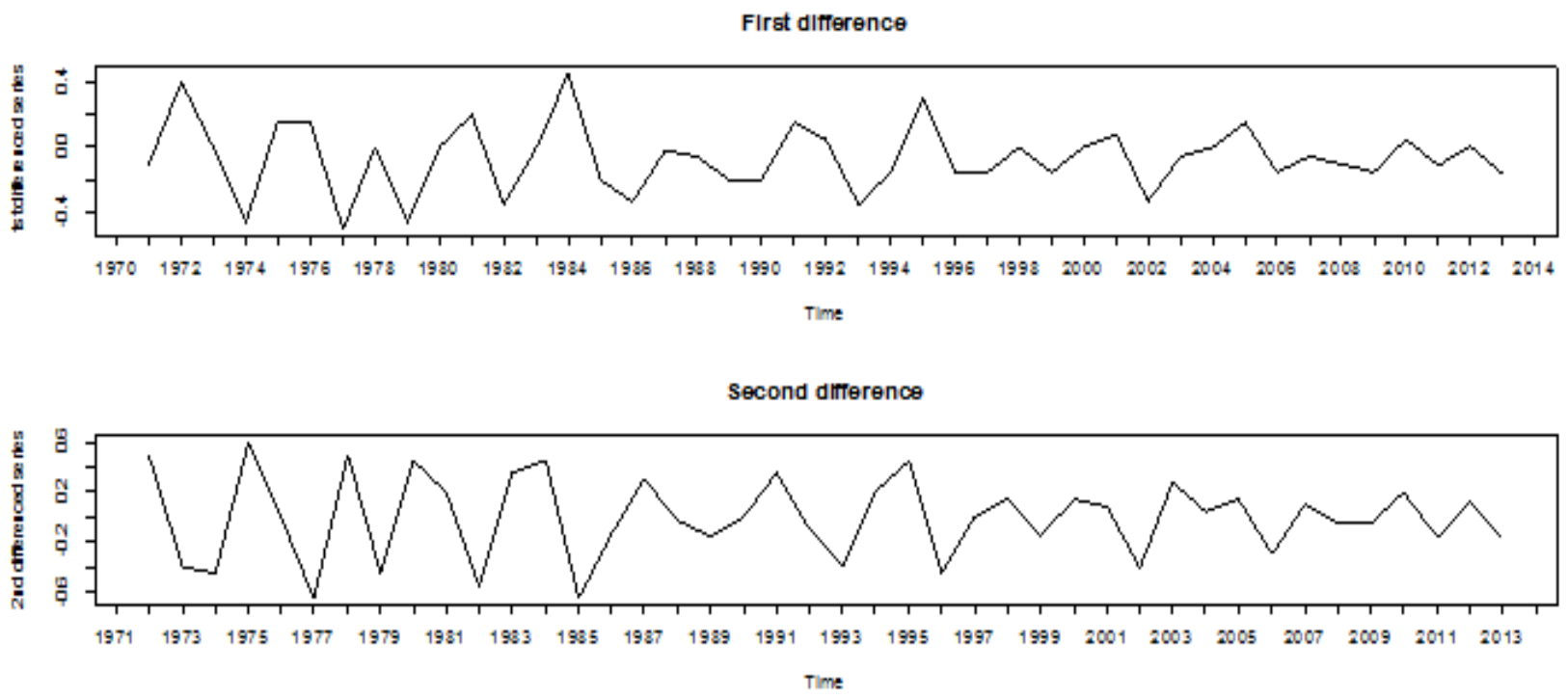

Fig 2(c):- $1^{\text {st }}$ difference and $2^{\text {nd }}$ difference series plotting

The autocorrelation function (ACF) for the differenced data (Figure 2(b)) diminishes quickly indicating an autoregressive (AR) model. The PACF also dies down after 2 significant levels. Tentatively, different possible models with varying combination of parameters for the ARIMA $(\mathrm{p}, \mathrm{d}, \mathrm{q})$ is fitted and the Akaike's Information Criterion (AIC), the Akaike's bias corrected Information Criterion with a correction (AICC) and the Bayesian Information Criterion (BIC) are used to select the best fit model. The results are tabulated in Table 1(a). A comparison of the AIC, AICC and the BIC values for the possible models shows that ARIMA $(3,1,1)$ has the least AIC, AICc and the BIC values and is therefore selected as the best fit model for the data. YuleWalker equation matrix gives the coefficients of the parameters of the equation with their standard errors. 
ISSN No:-2456-2165

\begin{tabular}{|c|c|c|c|}
\hline Models & AIC & BIC & AICc \\
\hline AR(1) & -0.20 & 5.15 & -0.11 \\
\hline AR(2) & 1.12 & 8.25 & 1.41 \\
\hline AR(3) & -2.16 & 6.76 & -1.56 \\
\hline MA(1) & 71.37 & 76.72 & 48.46 \\
\hline MA(2) & 48.63 & 55.76 & -5.88 \\
\hline ARIMA(1,1,0) & -5.98 & -2.46 & -4.53 \\
\hline ARIMA(1,1,1) & -4.83 & 0.46 & -6.87 \\
\hline ARIMA(1,1,2) & -7.47 & -0.42 & -9.19 \\
\hline ARIMA(2,1,1) & -9.48 & -4.20 & -10.37 \\
\hline ARIMA(2,1,2) & -10.97 & -3.93 & -8.52 \\
\hline ARIMA(3,1,0) & -9.55 & -0.74 & -12.63 \\
\hline ARIMA(3,1,1) & -13.23 & -6.19 & -16.43 \\
\hline
\end{tabular}

Table 1(a):- Table of AIC, AICc and BIC for possible fitted model

The ARIMA(3,1,1) Model for the series is:

$$
\begin{aligned}
Y_{t}=1.6454 Y_{t-1} & -0.862 Y_{t-2}+0.7876 Y_{t-3}-0.5714 Y_{t-4} \\
+ & 0.989 Z_{t-1}
\end{aligned}
$$

The model accuracy is further checked using LjungBox test and ACF plots of the residuals. A diagnostic of the residuals by the ACF shows that the ACF values are all within the 5\% zero-bound - indicating that there is no correlation amongst the residuals. This plot is used as an indicator of the independence of the residual terms. The Ljung-Box test statistic with degree of freedom $(\mathrm{df})=1$ and p-value $=0.8692$ which is greater than the $5 \%$ level of significance does not reject the null hypothesis that the residuals are random and have no correlation.

Figure 2(d) shows the plot of the (observed) time series data from 1970 to 2013, which was used for the modelling. From the figure, it can be observed that 5-14 mortality rates in India were high in 1970 (i.e. 3.4 deaths per 1,000 live births), which decreased gradually over time to about 0.7 per 1,000 live births in 2013 . The portion of the figure from the year 2014 to 2017 shows the in-sample forecast values by the $\operatorname{ARIMA}(3,1,1)$ model produced from the data with the $95 \%$ Confidence Interval for each of the forecast value. The values are tabulated in Table 1(b).

\begin{tabular}{|c|c|c|c|}
\hline Year & $\begin{array}{c}\text { Observed } \\
\text { Value }\end{array}$ & $\begin{array}{c}\text { Forecast } \\
\text { Value }\end{array}$ & $\begin{array}{c}\text { Confidence } \\
\text { Interval }\end{array}$ \\
\hline $\mathbf{2 0 1 4}$ & 0.65 & 0.69 & $0.3-0.95$ \\
\hline $\mathbf{2 0 1 5}$ & 0.7 & 0.65 & $0.22-1.01$ \\
\hline $\mathbf{2 0 1 6}$ & 0.6 & 0.7 & $0.12-0.94$ \\
\hline $\mathbf{2 0 1 7}$ & 0.6 & 0.53 & $0.01-0.89$ \\
\hline
\end{tabular}

Table 1(b):- A 95\% Confidence Interval for the In-sample forecast values by $\operatorname{ARIMA}(3,1,1)$ model

In using Random Walk with Drift Model, due to the non-stationarity in the time series data (5-14 Mortality Rates for India), it is just as good to predict the change that occurs from one period to the next i.e. the quantity $Y_{t}-Y_{t-1}$, as to directly predict the level $Y_{t}$ of the series at each period. This is because the predicted change can always be added to the current level to yield a predicted level.

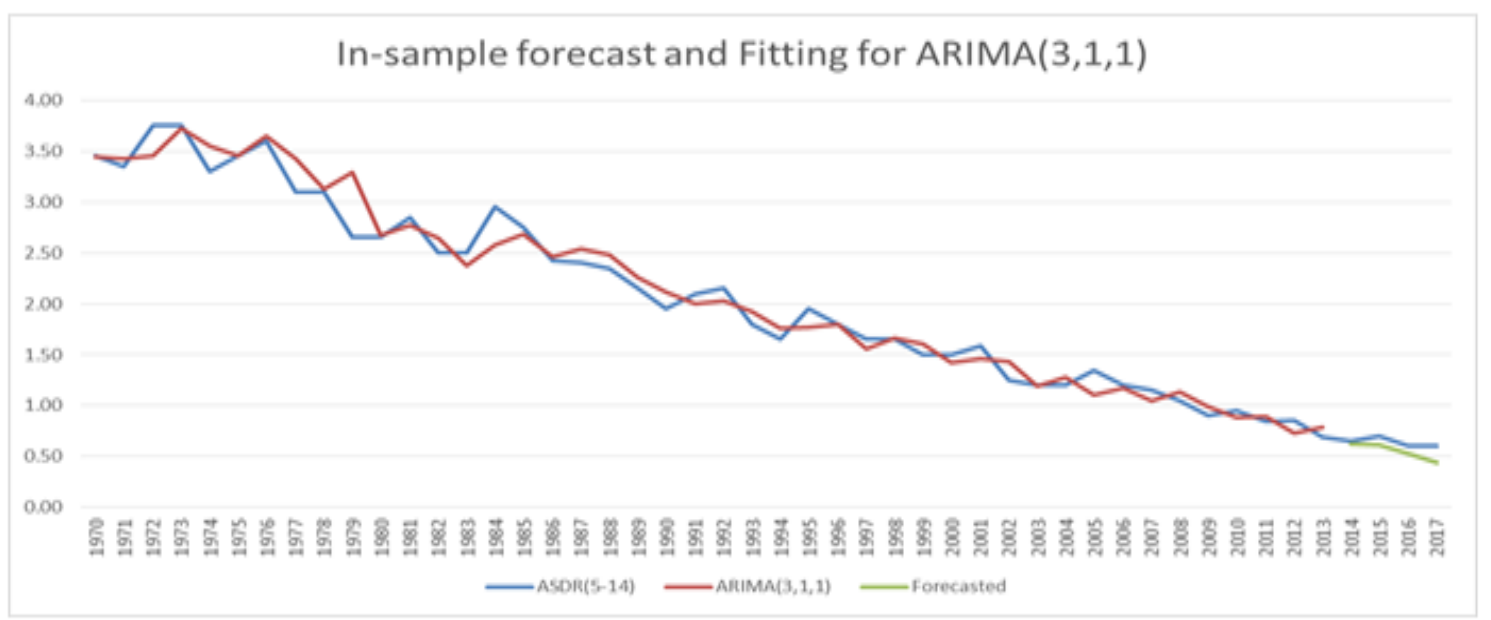

Fig 2(d):- In-sample forecast and fitting for $\operatorname{ARIMA}(3,1,1)$ 
1st difference series: $Y_{t}-Y_{t-1}=\alpha$ where $\alpha$ is the mean of the first differences known as the drift parameter.

This implies,

$$
Y_{t}=\alpha+Y_{t-1}
$$

The R statistical software was used for the analysis of this simple model, and below is the output from the analysis. The drift parameter $\alpha=-0.0642$ with Standard Error (s.e.) $=0.0317$. Thus, the Random Walk with drift model is:

$$
Y_{t}=-0.0642+Y_{t-1}
$$

The in-sample forecast values for Random Walk with drift model for the years 2013-17 are tabulated in Table 1(c). Figure 2(e) shows the plot for in-sample forecast using the Random Walk model.

\begin{tabular}{|c|c|c|c|}
\hline Year & $\begin{array}{c}\text { Observed } \\
\text { Value }\end{array}$ & $\begin{array}{c}\text { Forecast } \\
\text { Value }\end{array}$ & $\begin{array}{c}\text { Confidence } \\
\text { Interval }\end{array}$ \\
\hline $\mathbf{2 0 1 4}$ & 0.65 & 0.67 & $0.56-0.78$ \\
\hline $\mathbf{2 0 1 5}$ & 0.7 & 0.61 & $0.51-0.71$ \\
\hline $\mathbf{2 0 1 6}$ & 0.6 & 0.54 & $0.45-0.64$ \\
\hline $\mathbf{2 0 1 7}$ & 0.6 & 0.48 & $0.4-0.57$ \\
\hline
\end{tabular}

Table 1(c):- A 95\% Confidence Interval for the In Sample forecast Values by the Random Walk with Drift Model.

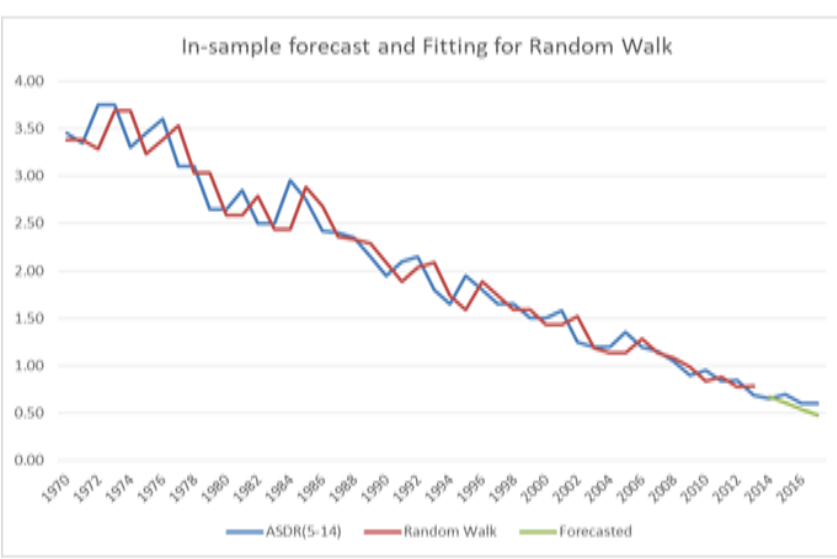

Fig 2(e):- In-sample forecast and fitting for Random Walk

After the in-sample forecast by each of the models, a forecast assessment was made to determine the best fit model among the two models. The visual inspection of each of the models shows that the in-sample forecast values of the Random Walk with drift model lie very close to those of the observed data values. A determination was therefore made between the ARIMA $(3,1,1)$ and the Random Walk with drift model, using the Mean Squared Error (MSE) and the Mean Absolute Percentage Error (MAPE) statistics, the results of which are shown in Table 1(d). The results of the forecast assessment for the two models show that, the $\operatorname{ARIMA}(3,1,1)$ produced comparatively lower values for both the MSE and the MAPE statistic. This indicates that the in-sample forecast values by the $\operatorname{ARIMA}(3,1,1)$ has lower deviations for the data values of that specified period. As a result, $\operatorname{ARIMA}(3,1,1)$ is selected as the best fit model for the 5-14 mortality rates of India and it is used to make the out-of sample forecasting for the years 2018-2025, represented in Table 2.

\begin{tabular}{|c|c|c|}
\hline Statistic & ARIMA (3,1,1) & Random Walk \\
\hline RMSE & 0.17 & 0.18 \\
\hline MAE & 0.12 & 0.14 \\
\hline MAPE & 6.37 & 6.73 \\
\hline MASE & 0.86 & 0.88 \\
\hline ACF1 & 0.02 & 0.24 \\
\hline
\end{tabular}

Table 1(d):- Results of Error Analysis of In-sample Forecast

\begin{tabular}{|c|c|c|}
\hline & \multicolumn{2}{|c|}{ Combined Population } \\
\hline \hline Year & ARIMA(3,1,1) & Random Walk \\
\hline $\mathbf{2 0 1 8}$ & 0.45 & 0.42 \\
\hline $\mathbf{2 0 1 9}$ & 0.39 & 0.36 \\
\hline $\mathbf{2 0 2 0}$ & 0.33 & 0.3 \\
\hline $\mathbf{2 0 2 1}$ & 0.27 & 0.24 \\
\hline $\mathbf{2 0 2 2}$ & 0.21 & 0.17 \\
\hline $\mathbf{2 0 2 3}$ & 0.15 & 0.11 \\
\hline $\mathbf{2 0 2 4}$ & 0.09 & 0.05 \\
\hline $\mathbf{2 0 2 5}$ & 0.03 & -0.01 \\
\hline
\end{tabular}

Table 2:- 95\% Confidence Interval for the out-of sample forecast up to 2025 using both $\operatorname{ARIMA}(3,1,1)$ and Random Walk with drift model for Combined Population

Based on the objectives and the analysis of the time series data at hand, a conclusion can be drawn that of the different parameters of ARIMA model that were tried in modelling and the Random Walk with drift model that was used $\operatorname{ARIMA}(3,1,1)$ is the best fit model and out-of sample forecasting has been done as per Table 2. The conclusion has been made that the mortality rate for India in the age group 5-14 will stand at 0.03 per 1000 live births by 2025 . Random Walk with drift model contrastingly predicts negative Mortality rate for males in 2025 of -0.01 which validates further in rejecting the model. This work has been extensively carried out and replicated for the same age group in the male and female population separately. The step wise procedure showed that $\operatorname{ARIMA}(3,1,1)$ is the best fit model for both male and female population thus giving a generalized time series model that is independent of the gender differentials and can be used for forecasting 
mortality trends and patterns in India. The predicted 5-14 mortality rate for males by $\operatorname{ARIMA}(3,1,1)$ model for 2025 is 0.09 per 1000 live births and that for females is 0.06 . Random Walk with drift model contrastingly predicts negative Mortality rate for males in 2025 of 0.33 which validates further in rejecting the model. Rate of decline of female mortality rates has been much faster and it stands at a lower index at present in comparison to males.

ARIMA model takes into consideration a weighted value of the data at previous time points summed with the weighted values of residuals in the past time due to some errors (white noise) which makes it very suitable to adapt to changing conditions in time. A generalized statement can thus be made that the time series model proposed for fitting is independent of the gender differentials and in context of the mortality trends and patterns in India this model can be used for forecasting.

\section{CONCLUSION AND SCOPE OF THE STUDY}

The study concentrates on modelling the mortality rates data using linear probabilistic time series models and doesn't take into consideration fitting non-linear models which might give better estimates. While $\operatorname{ARIMA}(3,1,1)$ can be seen to give the best results, it must also be noted that it does not take into account the socio-economic, climatic or political changes in the society, which also has a deep effect on the demographic parameters. Thus, beyond the extensive study, some more probe seems necessary.

The decline in the forecasted values of the 5-14 mortality rates for India by the $\operatorname{ARIMA}(3,1,1)$ model shows that the government's policies and health care interventions towards realization of the MDG4 goal are actually working positively. The study has mainly focused on the total population of India. This study should be extended to include the urban and rural population and to study the gender differences in the mortality patterns. Nonlinear time series models and hybrid models can be incorporated to design a general model which can be independent of gender, and type of residence as well.

\section{ACKNOWLEDGMENT}

The authors are thankful to Dr. Usha Ram, Department of Public Health and Mortality Studies, International Institute for Population Sciences, Mumbai, India for her encouragement and kind support. The help and guidance from her shall carry a long way in the journey of life which we are about to embark upon. The authors would also like to kindly acknowledge, the Office of The Registrar General and Census Commissioner, India, for sharing the data required for the study

\section{REFERENCES}

[1]. Ambalavanan, N., Carlo, W.A., Bobashev, G., Liu, B., Poole, K., Fanaroff, A. A.,Stoll, B.J., Ehrenkranz, R. and Wright, L. L. (2005): Prediction of Death for Extremely Low Birth Weight Neonates.

[2]. Amouzou, A., Richard, S. A., Friberg, I.K., Bryce, J., Baqui, A. H. and El Arifeen. (2010). "How well does LiST capture mortality by wealth quintile? A comparison of measured versus modelled mortality rates among children under-five in Bangladesh." International journal of Epidemiology (2010)39(suppl)

[3]. Arokiasamy, P., Yadav, S. (2014): " Changing age patterns of morbidity vis-à-vis mortality in India" J Biosoc Sci. 2014 Jul;46(4):462-79. doi: 10.1017/S002193201300062X. Epub 2013 Nov 8.

[4]. Barik, D., Desai, S. Vanneman, R. : “Adult Mortality in India: The Health-wealth Nexus"

[5]. Bisgaard, S., Kulachi, M. (2011).Time Series Analysis and Forecasting by Example. John Wiley \& sons, Inc. Hoboken, New Jersey.

[6]. Blot, S. Brusselaers, N., Monstrey, S., Vandewounde, K., De Waele, J.J., Colpaert, K., Decruyenaere, J.,Malbrain, M., Lafaire C., Fauville, J.P., Jennes, S., Casaer, M. P., Muller, J., Jacquemin, D., De Bacquer, D. and Hoste, E.(2009). "Development and validation of a model for prediction of mortality in patients with acute burn injury. The British journal of surgery.2009 Jan; 96(1):1117

[7]. Boschi-Pinto, C., Velebit, L. and Shibuya K. (2008)."Estimating Child Mortality due to diarrhea in developing in developing countries." Bulletin of the World Health Organization 2008;86: 710-717

[8]. Broughton, S.J., Berry, A., Jacobe, S.,Cheeseman, P. and Tarnow-Mordi, W.O. (2004). "The Mortality Index for Neonatal Transportation Score." A new Mortality Prediction Model for Retrieved Neonates.

[9]. Chatfield, C. (1975). The Analysis of Time Series:An Introduction, Chapman and Hall/CRC.

[10]. Chartfield, C. (2000). Time Series Forecasting

[11]. Chaurasia, A. (1992) :" Regional variations in age pattern of mortality in India.” J Inst Econ Res. 1992 Jul;27(2):1-11.

[12]. Clark, A. W. (1987). "Social Demography of Excess Female Mortality in India: New Directions." Economic \& Political Weekly 22(17): WS12WS15+WS18-WS21. CPR (2011). India's Health Insurance Scheme for the Poor: Evidence from the Early Experience of the Rashtriya Swasthya Bima Yojana

[13]. Coghlan, Avril. A little book of R for Time Series.

[14]. Dyson, T. (1984). "Excess Male Mortality in India." Economic \& Political Weekly 19(10): 422-426.

[15]. Hussein, M.A.(1993). "Time Series analysis for forecasting both fertility and mortality levels in Egypt until year 2010." The Egyptian population and family planning review 1993 Dec;27(2):67-81. 
[16]. Kesarwani, R. (2016): Age Patterns of Mortality in India.

[17]. Klose, C., Pircher, M. , Sharma, S. (2004). Univariate Time Series Forecasting "UK Okonometrische Prognose"

[18]. Kumar, D. N. and Maity, R. (2008). Bayesian dynamic modelling for nonstationary hydroclimatic time series forecasting along with uncertainty quantification.

[19]. Liu, L., Johnson, H.L.,Cousens, S., Perin, J., Scott, S., Lawn, J.E., Rudan, I., Campbell, H., Cibulskis, R., Li, M., Mathers, C. and Black, R.E.(2012). Global. regional, and national causes of child mortality: an updated systematic analysis for 2010 with time trends since 2000

[20]. Masquelier, B., Hug, L., Sharrow, D., Hogan, D., Hill, K., Liu, J., Pedersen, J. and Alkema, L (2018): Global, regional, and national mortality trends in older children and young adolescents (5-14 years) from 1990 to 2016: an analysis of empirical data

[21]. Mojekwu, J.N., and Ajijola, L.A. (2011). Developing a model for estimating infant mortality rate of Nigeria.

[22]. Mohamed, I. E. (2008). Time Series Analysis Using SAS, Part 1, The Augmented Dickey-Fuller (ADF) Test.

[23]. Mahapatra, P. An Overview of the Sample Registration System in India, Institute of Health Systems, Hyderabad, AP500004

[24]. Mari Bhat, P.N.(2002). Completeness of India's Sample Registration System: An assessment using the general growth balance method (Population Studies, Vol. 56, No. 2 (Jul., 2002), pp. 119-134).

[25]. Navaneetham, K. (2011) : "Mortality Trends and Patterns in India: Historical and Contemporary Perspectives"

[26]. Opare, P.E.(2014). Time Series Models For The Decrease In Under-Five Mortality Rate In Ghana Case Study $1961-2012$

[27]. Pedroza, C.(2006). A Bayesian forecasting model: Predicting U.S. male mortality. Oxford journals, volume 7 ,issue 4. Pp 530-550

[28]. Preston, S. H. (1975). "The changing relation between mortality and level of economic development." Population studies 29(2): 231-248.

[29]. Preston, S. H. (1980). Causes and consequences of mortality declines in less developed countries during the twentieth century. Population and economic change in developing countries, University of Chicago Press: 289-360

[30]. Purwanto, Eswaran, C. and Logeswaran, R. (2010). "A Comparison of ARIMA, Neural Network and Linear Regression Models for predicting of Infant Mortality Rate.” Ams, pp 34-39, 2010. Fourth Asia International Conference on Mathematical/ Analytical Modelling and Computer Simulation, 2010.

[31]. Rao, C., Adair, T., and Kinfu, Y. (2010). Using Historical Vital Statistics to Predict the Distribution of Under-Five Mortality by cause.

[32]. Rastogi, P.K., Sreenivas, V. and Kumar, N. (2009). Validation of CRIB II for prediction of Mortality in Premature Babies. 87
[33]. Saikia, N., Singh, A and Ram, F. (2010): "Has Child Mortality in India Really Increased in the Last Two Decades?" Economic and Political Weekly.

[34]. Saikia, N., Bora, J.K., Luy, M (2019) : "Socioeconomic disparity in adult mortality in India: estimations using the orphanhood method" Genus volume 75, Article number: 7 (2019)

[35]. Sankrithi, U., Emanuel, I., and Van Belle, G. (1991). "Comparison of linear and exponential multivariate models for explaining national infant and child mortality." International journal of epidemiology.1991 Jun;20(2):565-70.

[36]. Siu-Hang Li, J., Hardy, M.R. and Tank, S. (2009). "Uncertainty in Mortality Forecasting: An Extension to the classical Lee-Carter Approach." The journal of the International Actuarial Association; volume 39, issue 01 .

[37]. West, M. and Harrison, J. (1997). Bayesian Forecasting and Dynamic Models, 2nd ed. Springer Series in Statistics. Springer-Verlag New- York, Inc. 